\title{
An Assay of Deoxyadenosine and Adenosine in Human Plasma by HPLC
}

\author{
Charles A. Koller, Philip L. Stetson, Louis D. Nichamin, \\ ANd BeVerly S. Mitchell
}

Simpson Memorial Research Institute, Department of Internal Medicine, and Upjohn Center for Clinical Pharmacology, University of Michigan, Ann Arbor, Michigan 48109

Received March 24, 1980

The altered metabolism of both deoxyadenosine (1) and adenosine (2) has been causally implicated in the severe combined immunodeficiency disease associated with congenital deficiency of the enzyme adenosine deaminase (adenosine aminohydrolase, EC 3.5.4.4, ADA). In this condition, the pathway of deamination of deoxyadenosine to deoxyinosine and adenosine to inosine is unavailable (3). In the presence of exogenous ADA inhibitors such as $2^{\prime}$-deoxycoformycin ( 2 '-DCF), adenosine inhibits growth of human lymphocytes in vitro, whereas deoxyadenosine results in cytotoxicity (4). Adenosine has been quantitated in human plasma and urine (5-8) but deoxyadenosine has to date been detected only in urine $(8,9)$. To better understand the roles of adenosine and deoxyadenosine under various conditions, we have developed a rapid method for their analyses in plasma. Following barium hydroxide and zinc sulfate treatment, which extracted the adenine nucleosides from plasma without degrading them, deoxyadenosine and adenosine were separated by reverse-phase high-pressure liquid chromatography (HPLC).

\section{MATERIALS AND METHODS}

$\left[\mathrm{U}-{ }^{14} \mathrm{C}\right]$ adenosine $(559 \mathrm{Ci} / \mathrm{mole})$ and $\left[\mathrm{U}-{ }^{14} \mathrm{C}\right]$ deoxyadenosine $(505 \mathrm{Ci} /$ mole) were obtained from Amersham Corporation (Arlington Heights, Ill.). The stock solutions were diluted 1:2000 (v/v) with water prior to use to give final concentrations of 45 and $25 \mathrm{~nm}$ of adenosine and deoxyadenosine, respectively. Nonradioactive adenosine, deoxyadenosine, inosine, and deoxyinosine and calf intestine ADA (2350 U/ml) were obtained from Sigma Chemical Company. 
Plasma extraction procedure. The extraction procedure used is a modification of the method of Somogyi (10) and was performed at room temperature. Duplicate $500 \mu$ l plasma samples were mixed with $1180 \mu$ l of the diluted $\left[{ }^{14} \mathrm{C}\right]$ adenosine or $\left[{ }^{14} \mathrm{C}\right]$ deoxyadenosine; $200 \mu$ were removed and analyzed for radioactivity. Then $200 \mu \mathrm{l}$ of $150 \mathrm{~mm} \mathrm{Ba}(\mathrm{OH})_{2}$ was added. After $1 \mathrm{~min}, 320 \mu \mathrm{l}$ of $150 \mathrm{~mm} \mathrm{ZnSO}_{4}$ was added to give a final volume of $2000 \mu \mathrm{l}$. The samples were vortexed, the precipitate was allowed to settle for $3 \mathrm{~min}$, and the tubes were centrifuged at $500 \mathrm{~g}$ for 3 min. The supernatant was carefully decanted and was suitable for analysis without further purification. In order to prepare the samples for peak identification as described below, $110-\mu \mathrm{l}$ aliquots of the sample were treated either with $10 \mu \mathrm{l}$ of $130 \mathrm{mM} \mathrm{Na}_{4}$ EDTA or with $10 \mu \mathrm{l}$ of ADA diluted 1:10 (v/v) with $130 \mathrm{mM} \mathrm{Na}_{4}$ EDTA. EDTA was used to chelate the free $\mathrm{Zn}^{2+}$ in the extract and completely reversed the inhibition of ADA by this ion: $200 \mu \mathrm{l}$ of the supernatant was also analyzed for radioactivity.

Recovery of the labeled nucleosides was quantitative and no quench corrections were necessary.

Adenosine and deoxyadenosine were quantitated by high-pressure liquid chromatography using solvent delivery pumps (Model $6000 \mathrm{~A}$ ), double beam uv detector operating at a fixed wavelength of $254 \mathrm{~nm}$ (Model 440), and reverse-phase $\left(\mu\right.$ Bondapak $\left.\mathrm{C}_{18}, 30 \mathrm{~cm} \times 4 \mathrm{~mm}\right)$ column fron Waters Associates (Milford, Mass.). Separation of the nucleosides in $100 \mu \mathrm{l}$ of sample was accomplished at ambient temperature by isocratic elution using a solvent composed of $50 \mathrm{mM} \mathrm{KH_{2 }} \mathrm{PO}_{4}-10 \%$ methanol $(\mathrm{v} / \mathrm{v}), \mathrm{pH}$ 4.5. The flow rate was $2.0 \mathrm{ml} / \mathrm{min}$, resulting in a back-pressure of approximately $2900 \mathrm{psi}$. Fractions were collected at specified times after passing the detector and were analyzed for radioactivity.

\section{RESULTS}

The separation of adenosine and deoxyadenosine and their deamination products inosine and deoxyinosine is shown in Fig. 1. In the range of $0-10$ $\mu \mathrm{g}$ per injection, the peak uv absorbance of the adenine nucleosides increased linearly with the amount injected (Fig. 2). The lower limit of detection for adenosine and deoxyadenosine using this method was approximately $100 \mathrm{~nm}$. Following extraction, samples were stable for greater than 2 weeks at $4^{\circ} \mathrm{C}$.

Application. We studied plasma samples from patients being treated with the ADA inhibitor 2 '-deoxycoformycin. Following inhibition of systemic ADA activity in one representative patient, both adenosine and deoxyadenosine were readily detected (Fig. 3a). Adenosine, but not deoxyadenosine, could also be detected using standard perchloric or trichloroacetic acid extractions (11), although some samples still retained unidentified compounds which obscured analysis (data not shown). 

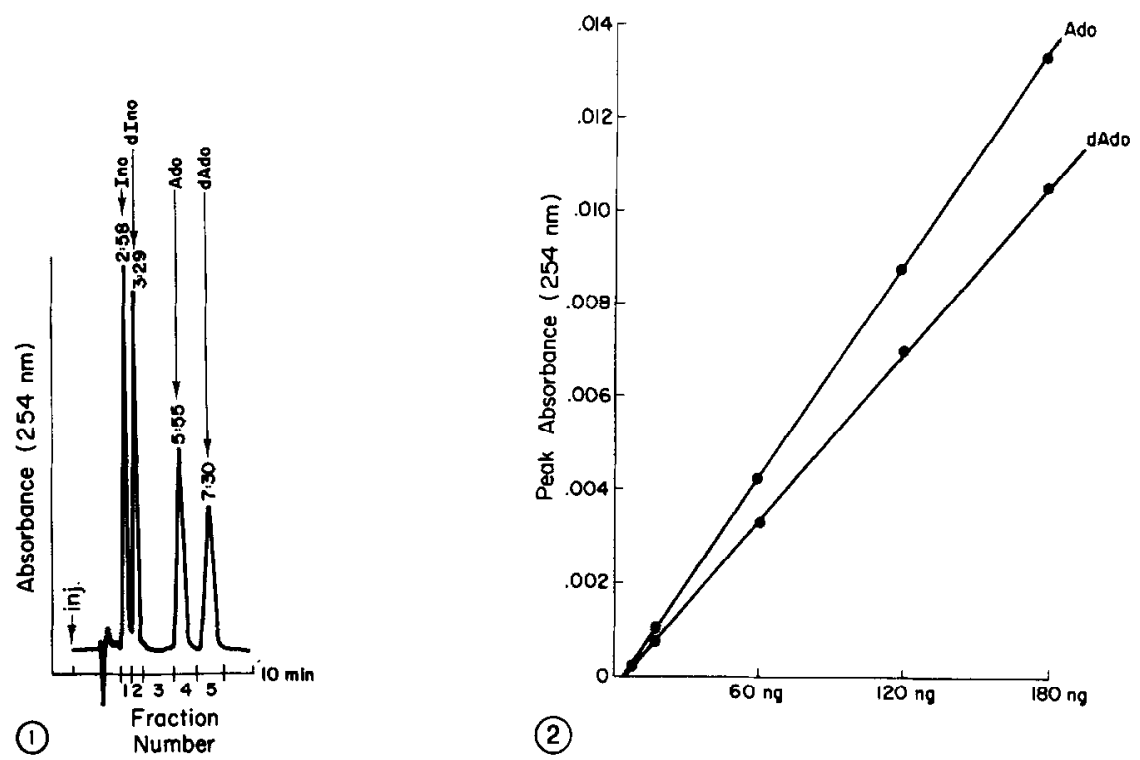

FIG. 1. Separation of inosine, deoxyinosine, adenosine, and deoxyadenosine ( $26.7 \mathrm{ng}$ of each in water) using HPLC as described under Materials and Methods. Times from injection to peaks are noted in min:sec. Fractions were collected at the following intervals: fraction 1 (inosine), 2:50-3:20; fraction 2 (deoxyinosine), 3:20-3:55; fraction 3 (a middle fraction) 3:55-5:40; fraction 4 (adenosine), 5:40-6:50; and fraction 5 (deoxyadenosine), 6:50-8:15.

FIG. 2. Peak absorbance at $254 \mathrm{~nm}$ vs. amount of adenosine or deoxyadenosine injected.

For definitive identification of the peaks with the retention times of adenosine and deoxyadenosine, we used the enzymatic peak shift technique. The enzyme adenosine deaminase converts adenosine and deoxyadenosine to inosine and deoxyinosine, respectively. Thus, both the adenosine and deoxyadenosine peaks will move on the chromatogram following deamination. After the sample shown above in Fig. 3a was treated with ADA, we obtained the chromatogram shown in Fig. 3B. The absorbance in fractions 4 and 5 decreased markedly between Figs. 3a and $b$, because the adenosine (fraction 4 ) and deoxyadenosine (fraction 5) had been deaminated to inosine (fraction 1) and deoxyinosine (fraction 2), respectively. We were able to confirm that deamination was complete by demonstrating an appropriate shift in radioactivity in ADA-treated samples containing the radiolabeled adenosine or deoxyadenosine internal standard (Table 1). Using the radioactive peak shift, complete deamination was confirmed in all samples following ADA treatment.

Figure 4 shows plasma deoxyadenosine and adenosine levels for 8 days following deoxycoformycin therapy in the patient referred to above. This complex pattern of plasma adenine nucleosides represents the net balance 
TABLE 1

Enzymatic Shift of Radiolabeled Adenosine and DEOXYADENOSINE INTERNAL STANDARDS

\begin{tabular}{|c|c|c|c|c|c|}
\hline \multirow{2}{*}{$\begin{array}{l}\text { Internal } \\
\text { standard }\end{array}$} & \multicolumn{5}{|c|}{ Fraction $^{u}$} \\
\hline & 1 & 2 & 3 & 4 & 5 \\
\hline$\left[{ }^{14} \mathrm{C}\right]$ Ado & & & cpm & & \\
\hline Before ADA & 40 & 45 & 38 & 1738 & 52 \\
\hline After ADA & 1704 & 49 & 43 & 36 & 28 \\
\hline \multicolumn{6}{|l|}{$\left[{ }^{14} \mathrm{C}\right] \mathrm{dAdo}$} \\
\hline Before ADA & 46 & 112 & 40 & 29 & 990 \\
\hline After ADA & 33 & 1016 & 37 & 32 & 35 \\
\hline
\end{tabular}

${ }^{a}$ Fractions correspond to those shown in Fig. 1.

between de novo production of nucleosides, degradation of nucleic acids, and altered metabolism and excretion subsequent to the drug-induced block in nucleoside degradation via ADA.

\section{DISCUSSION}

Deoxyadenosine is a direct precursor of dATP, the nucleotide which accumulates in and is presumably toxic to lymphoid cells in congenital
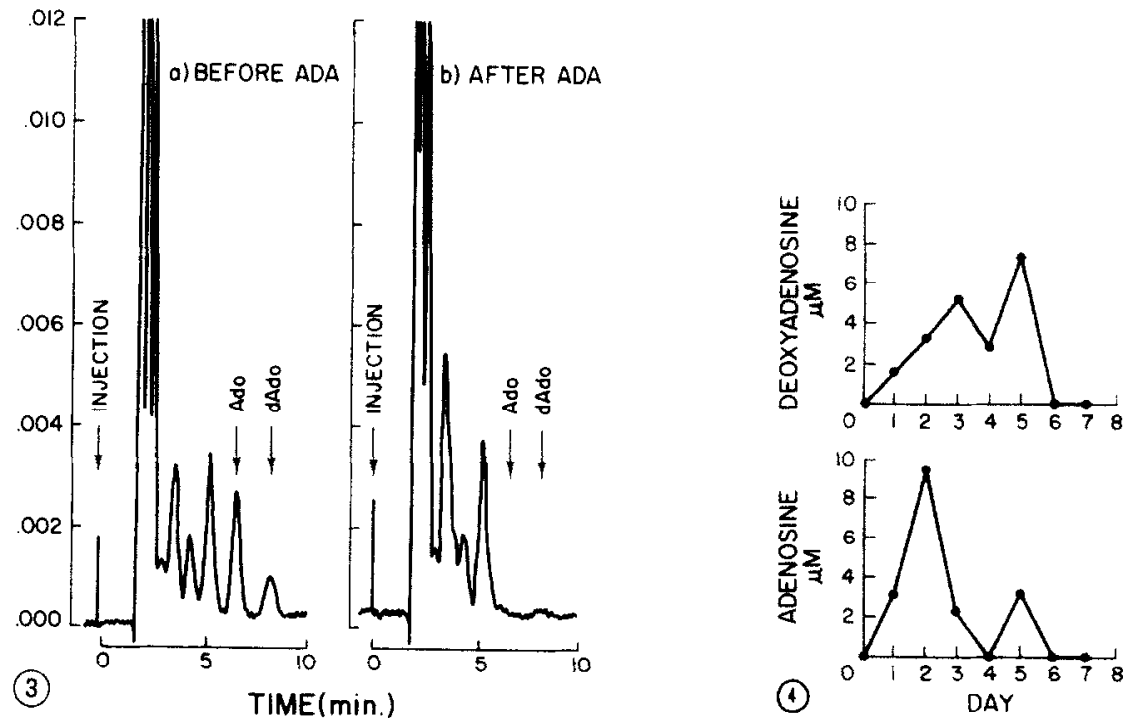

Fig. 3. (a) Chromatogram of extracted plasma in a patient being treated with an adenosine deaminase inhibitor. (b) Sample treated with exogenous adenosine deaminase for enzymatic peak shift identification. Extraction of adenosine $98.7 \%$. deoxyadenosine $98.6 \%$.

FIG. 4. Composite plasma deoxyadenosine (top) and adenosine (bottom) for 8 days following systemic inhibition of ADA. Patient treated with $1 \mathrm{mg} / \mathrm{kg} 2^{\prime}$-deoxycoformycin on Day 0. 
ADA deficiency. Thus, the most direct measure of the adequacy of enzyme replacement therapy (12) in this disease may be the determination of plasma deoxyadenosine levels. Not until deoxyadenosine is lowered to a nontoxic concentration could one expect a therapeutic response to this mode of intervention. Similarly, when toxicity to lymphoid cells is the goal of therapy, as in the treatment of acute leukemia with the ADA inhibitor 2'-deoxycoformycin, an increment in plasma deoxyadenosine levels should correlate with the therapeutic response (13). Therefore, a simple deoxyadenosine and adenosine assay is presently of therapeutic importance in clinical medicine.

This analysis of plasma deoxyadenosine and adenosine using reversephase HPLC offers features which are similar to methods previously used (5-8). Rapid analysis can be achieved with sensitivity and specificity. The results are quantitative and other uv-absorbing constituents of plasma do not interfere with the assay. Minimal sample preparation is required prior to chromatography, and since an isocratic elution mode was used, there is no delay between analyses. Thus, plasma can be prepared for HPLC within $10 \mathrm{~min}$ and adenosine and deoxyadenosine levels determined and confirmed within an hour. Serum samples are equally easy to assay.

The advantages of this method over a similar method for the analysis of adenosine (7) are twofold. First, the recovery of each nucleoside from the extraction procedure can be calculated on each sample by means of the radiolabeled internal standard. Second, the completeness of deamination can be confirmed for each peak. Confirmation of complete deamination is important for the measurement of deoxyadenosine and adenosine in the presence of ADA inhibitors such as $2^{\prime}$-deoxycoformycin or zinc. Incomplete deamination will cause an underestimation of nucleoside levels.

This method offers a major additional advantage over those previously suggested for the determination of plasma deoxyadenosine $(7,8)$, in that it avoids acid extraction while providing a clear supernatant. Deoxyadenosine is extremely labile at low $\mathrm{pH}$ (14-17), and decompcses to adenine and deoxyribose after acid extraction. Therefore, it is not surprising that previous attempts to detect deoxyadenosine in the plasma of ADA-deficient patients yielded undetectable levels (8). This method should greatly facilitate the measurement of deoxyadenosine and adenosine in plasma so that therapeutic manipulations involving these nucleosides can be more easily accomplished.

\section{SUMMARY}

We have developed a method for the simultaneous measurement of deoxyadenosine and adenosine in human plasma. A barium hydroxidezinc sulfate extraction procedure circumvented acid precipitation and preserved the deoxyadenosine. Deoxyadenosine and adenosine were separated using reverse-phase high-pressure liquid chromatography and 
their identities were confirmed by the enzymatic peak shift technique. A radioactive internal standard was used to measure extraction efficiency and to prove completeness of enzyme treatment for the peak shift technique. This assay is sensitive to $100 \mathrm{~nm}$ of either nucleoside in plasma and can be completed within $1 \mathrm{hr}$. This technique can be used to quantitate plasma deoxyadenosine and adenosine in patients with congenital adenosine deaminase deficiency or in patients being treated with ADA inhibitors.

\section{ACKNOWLEDGMENTS}

We would like to thank the Michigan Children's Leukemia Foundation for their support. B.S.M. is the recipient of an NIH Clinical Investigation Award 5K08 AM00442.

\section{REFERENCES}

1. Carson, D. A., Kaye, J., and Seegmiller, J. E., Proc. Nat. Acad. Sci. USA 74, 5677 (1977).

2. Hirshhorn, R., Fed. Proc. 36, 2166 (1977).

3. Meuwissen, H. J., Pollara, B., and Pickering, R. J., J. Pediatr. 86, 169 (1975).

4. Mitchell, B. S., Mejias, E., Daddona, P. E., and Kelley, W. N., Proc. Nat. Acad. Sci. USA 75, 5011 (1978).

5. Krstulovic, A. M., Brown, P. R., and Rone, D. M., Anal. Chem. 49, 2237 (1977).

6. Brown, P. R., Bobick, S., Heinley. F. L., J. Chromatogr. 99, 587 (1974).

7. Hartwick, R. A., and Brown, P. R., J. Chromatogr. 143, 383 (1977).

8. Kuttesch. J. F.. Schmalstieg, F. C., and Nelson, J. A. . J. Liq. Chromatogr. 1, 97 (1978).

9. Hirshhorn, R., Roegner, V., Jenkins, T., Seaman, C., Piomelli, S., and Borkowsky, W.. J. Clin. Invest. 64, 1130 (1979).

10. Somogyi, M., J. Biol. Chem. 160, 69 (1945).

11. Mollering, H., and Bergmeyer, H. U., in "Adenosine, Methods of Enzymatic Analysis" (H. U. Bergmeyer, Ed.), p. 491. Academic Press, New York, 1965.

12. Polmar, S. H., Stern, R. C., Schwartz, A. L., Wetzler, E. M., Chase. P. A., and Hirshhorn, R., N. Engl. J. Med. 295, 1337 (1976).

13. Koller, C. A., Mitchell, B. S., Grever, M. R., Mejias, E., Malspeis. L., and Metz, E. N., Cancer Treat. Rep. 63, 1949 (1979).

14. Levene, P. A., and London, E. S.. J. Biol. Chem. 81, 711 (1929).

15. Levene, P. A., and London, E. S., J. Biol. Chem. 83, 793 (1929).

16. Levene, P. A., and Mori, T., J. Biol. Chem. 83, 803 (1929).

17. Ulbricht, T. L. V., in "Comprehensive Biochemistry" (M. Florkin and E. H. Stotz, Eds.), Vol. 8, p. 186. Elsevier, New York, 1963. 